\title{
Postoperative Expansion of Dural Sac Cross-Sectional Area after Unilateral Laminotomy for Bilateral Decompression: Correlation with Clinical Symptoms
}

\author{
Seok-Won Chung, ${ }^{1}$ Min-Soo Kang', Yong-Hwan Shin', Oon-Ki Baek', Sang-Ho Lee ${ }^{2}$ \\ ${ }^{I}$ Department of Neurosurgery, Daegu Wooridul Spine Hospital, Daegu, \\ ${ }^{2}$ Department of Neurosurgery, Wooridul Spine Hospital, Seoul, Korea
}

Objective: Dural sac cross-sectional area (DSCSA) is a way to measure the degree of central spinal canal compression. The objective was to investigate the correlation between the expansion ratio of DSCSA after unilateral laminotomy for bilateral decompression (ULBD) and the clinical results for lumbar spinal stenosis.

Methods: We retrospectively reviewed the clinical data and radiographs of 103 patients who underwent ULBD for symptomatic spinal stenosis in one year. We compared preoperative and postoperative clinical data and DSCSA and evaluated the correlation between clinical and radiographic measurements.

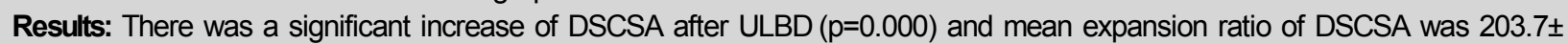
$147.2 \%$ (range $-32.9-826.1 \%$ ). Clinical outcomes, measured by VAS and ODI were improved significantly not only in early postoperative period, but also in the last follow-up. However, there were no statistically significant correlations between the preoperative DSCSA and clinical symptoms, Perioperative expansion ratio of DSCSA and clinical parameters were also not correlated to the improvement of clinical symptoms significantly in both early postoperative phase and last follow-up. Conclusion: Our result indicates that the DSCSA itself has a definite limitation to be correlated to the clinical symptoms, and thus meticulous correlation between the clinical presentation and MRI imaging is essential in determination of surgical treatment.

Key Words: Spinal stenosis $\cdot$ Minimally invasive spinal surgery $\cdot$ Laminotomy $\cdot$ Radiculopathy

\section{INTRODUCTION}

Degenerative lumbar spinal stenosis (DLSS) is defined as a narrowing of the lumbar spinal canal, lateral nerve root canals or the intervertebral neural foramina by patho-anatomic changes involving bulging disc, hypertrophy of the ligamentum flavum and facet-joints, which causes mechanical compression of spinal nerve roots. As the population grows older, DLSS is becoming increasingly prevalent and surgery for DLSS is the procedure most frequent performed in the adult spine ${ }^{4}$. In patients with symptomatic DLSS, the symptoms are most

\footnotetext{
- Received: June 27, 2014 - Revised: August 10, 2014

- Accepted: August 13, 2014

Corresponding Author: Min-Soo Kang, MD, PhD

Department of Neurosurgery, Daegu Wooridul Spine Hospital, 648

Gukchaebosang-ro, Jung-gu, Daegu 700-732, Korea

Tel: +82-53-212-3000, Fax: + 82-53-212-3068

E-mail: mansiki@hanmail.net

*This study was supported by a grant from the Wooridul Spine Foundation.

@This is an Open Access article distributed under the terms of the Creative

Commons Attribution Non-Commercial License (http://creativecommons.org/

licenses/by-nc/3.0/) which permits unrestricted non-commercial use, distribution,

and reproduction in any medium, provided the original work is properly cited.
}

commonly neurologic claudication, or low back pain, or sciatic pain. Despite the non-surgical treatment modalities including medication, physical therapy, bracing and epidural injections are advocated in some patients who experience mild symptoms or with poor general health conditions, it is well documented that the natural course of DLSS is often poor and many patients receive surgical treatment after all ${ }^{1,2}$. Traditionally, total laminectomy with or without fusion had been the standard operative techniques, but less invasive operative options such as the bilateral laminotomy and the unilateral laminotomy for bilateral decompression (ULBD) have been introduced and widely used during the past years. Especially, ULBD has been successfully used and subsequently modified to the adequate microsurgical technique for decompression of DLSS that minimizes operative invasiveness and tissue trauma while maximizing preservation of the spinal integrity and stability ${ }^{3,5,7,11,14,18,21)}$.

Dural sac cross-sectional area (DSCSA) is a way to measure the degree of central spinal canal compression ${ }^{15}$. To date, many studies have analyzed the relationship between DSCSA and the preoperative clinical symptoms, but it is still controversial $^{10,18,23)}$. Only a few studies have evaluated serial changes of DSCSA after posterior decompression for DLSS and to our knowledge, it is not published any studies that compare pre- 
operative and postoperative DSCSA after ULBD ${ }^{6,12,22)}$.

The main purpose of this retrospective study was to investigate the correlation between the expansion ratio of dural sac CSA after ULBD and the clinical results.

\section{MATERIALS AND METHODS}

\section{Patients}

Between June, 2010 and May, 2011, a total of 103 consecutive patients underwent single level ULBD at our hospital for symptomatic DLSS including 50 men (48.5\%) and 53 women (51.5\%). The age distribution ranges from 36 to 84 years, with a mean age at surgery of 61.0 years. Mean duration of symptom was 21.5 months (range, 2-120 months), and mean follow-up duration was 17.7 months (range, 13-34 months). Patient demographics are summarized in Table 1 and distribution of affected level is summarized in Table 2. The diagnosis was made on the basis of clinical symptoms, neurological examinations, and imaging studies including pain radiography, computed tomography (CT), MR myelography, and MRI of the lumbar spine. Patients with history of previous lumbar spinal surgery at affected or adjacent level, severe osteoporosis, polyneuropathy, spinal anomalies, and lower extremity peripheral arterial disease were excluded.

\section{Surgical Procedure}

The microsurgical ULBD, as previous described in detail, was performed in all instances ${ }^{19,20)}$. We choose an approach through the direction of more dominant symptoms were. After the correct level was confirmed based on the lateral radiograph, a vertical skin incision below $3 \mathrm{~cm}$ was made and routine muscular dissection performed to expose the lamina and facet to be operated. After ipsilateral partial hemilaminectomy and foraminotomy, the base of the spinous process was removed with a burr drill by medial angulation of the operative microscope to better visualize the contralateral side. The contralateral hemilaminae together with the hypertrophied medial

Table 1. Patient demographics

\begin{tabular}{|c|c|c|}
\hline \multicolumn{3}{|c|}{ Variables } \\
\hline \multicolumn{2}{|c|}{ No. of Patients } & 103 \\
\hline \multirow{2}{*}{ Gender } & Male & $50(48.5 \%)$ \\
\hline & Female & $53(51.5 \%)$ \\
\hline \multicolumn{2}{|c|}{ Age at operation in years (mean $\pm S D$ ) } & $61.0 \pm 10.8$ \\
\hline \multicolumn{2}{|c|}{ Symptom duration in months (mean $\pm S D$ ) } & $21.5 \pm 19.5$ \\
\hline \multicolumn{2}{|c|}{ Follow-up duration in months (mean $\pm S D$ ) } & $17.7 \pm 5.4$ \\
\hline
\end{tabular}

facet were partially removed and bilateral flavectomy was performed. After the decompression of contralateral lateral recess and neural foramina, the incision was closed. Care was taken to preserve the hypertrophied ligamentum flavum as long as possible for protection of the dural sac and nerve root during drilling. In 18 cases, discectomy was also performed.

\section{Clinical Methods}

At clinical examination, the following information of the clinical symptoms was registered. The duration of symptoms (in months), as reported by the patients, was noted and everyday functional disability was measured using the Oswestry Disability Index (ODI). The ODI questionnaire deals with problems caused by pain, and includes personal care, mobility, gender, social life, etc. Pain was measured with a linear visual analogue scale (VAS), in which the patient estimates the severity of the pain on a linear scale.

\section{Radiologic Methods}

In our hospital, routine postoperative MRI was checked within 3 days after ULBD surgery. MRI was performed by using a 1.5-T system (Magnetom Symphony; Siemens, Erlangen, Germany) using a spine-phased array coil. The imaging tests were performed with patients in the supine position with their hips slightly flexed. Scans performed by at the facet joint level at each intervertebral space were selected because this level is the most commonly involved in DLSS ${ }^{15,16}$. To evaluate the change of DSCSA, two spinal neurosurgeons separately measured the preoperative and postoperative DSCSA using a picture archiving communication system (PACS) feature (PiViewSTAR, INFINITT Healthcare Co, Seoul, Korea). The DSCSA was determined three times at separated occasions and the average of these 6 measurements was calculated to accommodate investigator error in assessing the margin of dural sac. The preoperative and postoperative DSCSAs were measured at the same site (Fig. 1).

Table 2. Distribution of spinal levels affected lumbar spinal stenosis and ULBD surgery.

\begin{tabular}{|c|c|c|}
\hline Level & No. of ULBDs $(n=103)$ & \% of ULBDs \\
\hline LI-2 & 2 & 1.9 \\
\hline L2-3 & 3 & 2.9 \\
\hline L3-4 & 9 & 8.7 \\
\hline$\lfloor 4-5$ & 86 & 83.5 \\
\hline L5-SI & 3 & 2.9 \\
\hline
\end{tabular}

ULBD, unilateral laminotomy for bilateral decompression 


\section{Statistical Analysis}

Statistical analysis relied on standard software (SPSS v16.0 for Windows, SPSS, Inc, Chicago, IL, USA). Paired t-tests were used to compare the differences between pre- and post-operative DSCSA values. To compare the interval changes of VAS and ODI after ULBD, repeated ANOVA test was performed. In addition, the results of VAS, ODI and DSCSA were correlated using the Spearman correlation matrix. Statistical significance was set at $\mathrm{p}<0.05$.

\section{RESULTS}

\section{Perioperative Interval Change of Dural Sac Cross Sectional Area}

The mean DSCSA preoperatively and in the early postopera-
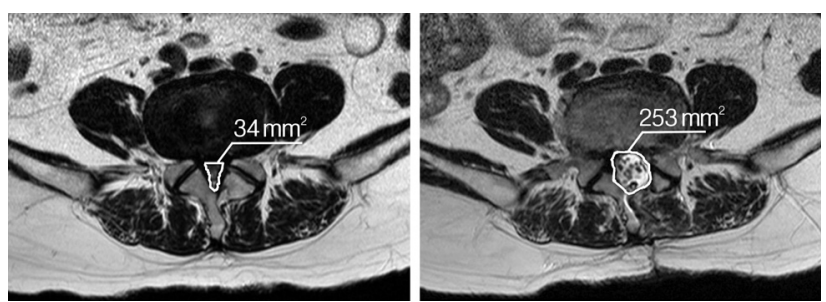

Fig. 1. Illustrations of the dural sac cross-sectional area measurement technique. (A) There is narrowing of spinal canal caused by bulging of the disc, hypertrophy of ligmentum flavum, and facet arthrosis with a dural sac cross-sectional area of $34 \mathrm{~mm}^{2}$ on L4-5 level. (B) After operation of ULBD via left side approach, dural sac cross-sectional area at the same site was increased to $253 \mathrm{~mm}^{2}$. ULBD, unilateral laminotomy for bilateral decompression.

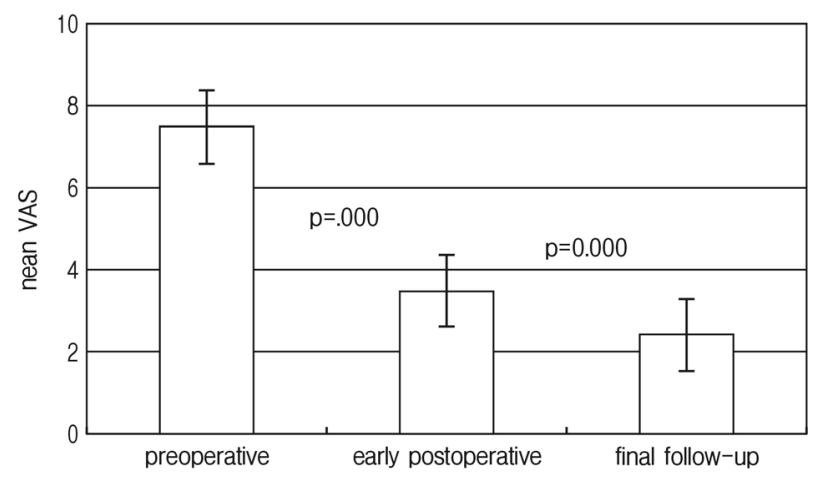

Fig. 2. Mean visual analogue scale preoperatively, in the early postoperatively and on last follow-up. The score decreased significantly between the preoperative and early postoperative phases $(p=0.000)$ and between the early postoperative phase and on last follow-up $(p=0.000)$. tive phase was $60.7 \pm 24.7 \mathrm{~mm}^{2}$ (mean \pm standard error) (range 19-134 $\mathrm{mm}^{2}$ ), $161.0 \pm 46.9 \mathrm{~mm}^{2}$ (range $47-270 \mathrm{~mm}^{2}$ ). There was a significant increase of DSCSA after ULBD $(p=0.000)$. The mean expansion ratio of DSCSA after ULBD was 203.7 \pm $147.2 \%$ (range $-32.9-826.1 \%$ ). The patient's age at surgery was not correlated with the expansion ratio of DSCSA ( $p=$ $0.059)$, and the duration of symptom was also not correlated $(p=0.656)$.

\section{Clinical outcomes}

The mean VAS score preoperatively, at the time of postoperative MRI acquired (within 3 days postoperatively), and at the last follow-up was $7.5 \pm 0.9$ (range 4-10), 3.5 \pm 0.9 (range $2-5$ ), and 2.4 \pm 0.9 (range 1-4), respectively. Not only in the early postoperative phase, but also in the last follow-up, VAS score after ULBD was improved, and it was statistically significant each other $(p=0.000)$ (Fig. 2).

ODI was also improved in the similar pattern of VAS after ULBD. The mean ODI preoperatively, at the time of postoperative MRI acquired, and at the last follow-up was 58.6 $\pm 7.8 \%$ (range 32.0-82.0\%), 32.1 $\pm 6.6 \%$ (range 16.0-44.0\%), and 23.8 $\pm 8.1 \%$ (range 11.0-40.0), respectively, and in was also statistically significant each other $(\mathrm{p}=0.000)$ (Fig. 3). In all cases, clinical parameters (VAS and ODI) were improved after ULBD. However $6(5.8 \%)$ patients showed no more improvement between early postoperative phase and last follow-up.

\section{Correlation Between Radiologic Findings and Clinical Symptoms}

There were no statistically significant correlations between the preoperative DSCSA and clinical symptoms measured by

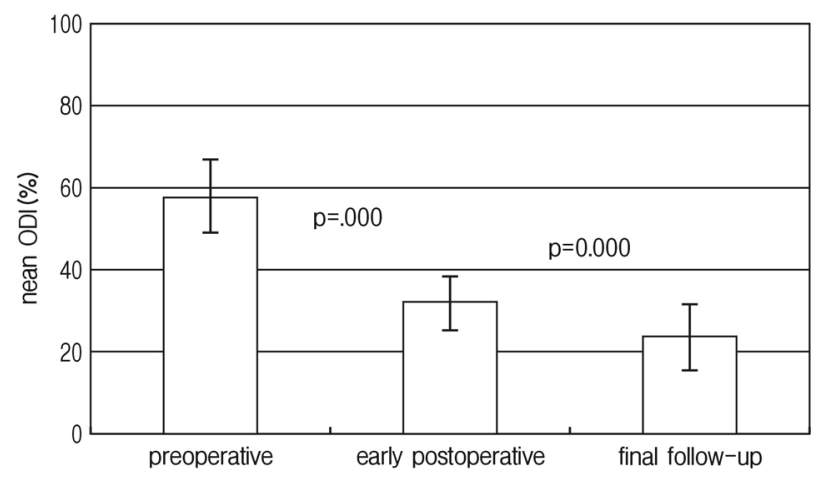

Fig. 3. Mean Oswestry disability index preoperatively, in the early postoperatively and on last follow-up. The percentile score decreased significantly between the preoperative and early postoperative phases $(p=0.000)$ and between the early postoperative phase and on last follow-up $(p=0.000)$. 
Table 3. Correlation between radiologic findings and clinical symptoms

\begin{tabular}{lrl}
\hline \hline \multicolumn{2}{c}{ Correlation Coefficient } & p-value \\
\hline Preoperative DSCSA $\left(\mathrm{mm}^{2}\right)$ & -0.118 & 0.234 \\
VAS & -0.082 & 0.410 \\
ODI (\%) & \\
Expansion ratio of DSCSA (\%) & 0.803 \\
VAS (Pre-Early) & -0.025 & 0.344 \\
VAS (Pre-Last) & -0.094 & 0.420 \\
ODI (Pre-Early) & -0.080 & 0.229 \\
ODI (Pre-Last) & -0.120 &
\end{tabular}

DSCSA, dural sac cross-sectional area

VAS, visual analogue sclale; ODI, Oswestry disability index

Pre, preoperative; Early, early postoperative; Last, last follow-up

preoperative VAS $(\mathrm{r}=-0.118 ; \mathrm{p}=0.234)$ and ODI $(\mathrm{r}=-0.082$; $\mathrm{p}=0.410$ ).

Despite both the DSCSA and clinical symptoms were improved after operation, comparing perioperative expansion ratio of DSCSA and clinical parameters, there was no statistically significant correlation. The expansion ratio of DSCSA was not correlated to the improvement of VAS score significantly in both early postoperative phas $(r=-0.025 ; \mathrm{p}=0.803)$ and last follow-up ( $r=-0.094 ; p=0.344)$. It was also not correlated to the improvement of ODI in early postoperative phase $(r=-0.080 ; p=0.420)$ and last follow-up $(r=-0.120 ; p=0.229)$. Table 3 summarized the correlation between radiologic findings and clinical symptoms.

\section{DISCUSSION}

The main object of the surgical treatment for DLSS is to significantly increase the spinal canal diameter by removal of ligamentum flavum, the lamina and by performing a partial medial facetectomy, and thereby improving the patients' symptoms. In many previous published articles, the DSCSA was measured to evaluate the severity of spinal stenosis ${ }^{8,9,10}$, 13,17,18,23). However, the relationship between the DSCSA and the severity of preoperative symptoms has been uncertain, and showed a poor correlation in most of published studies. Lohman et al. reported that the severity of the clinical symptoms of spinal stenosis does not significantly correlates with the DSCSA estimated on CT images ${ }^{9}$. Sirvanci et al. showed no significant correlation between the dural sac CSA and ODI percentage score ${ }^{18)}$. Sigmundsson et al. expanded the questionnaires for EQ-5D, SF-36, and walking distance and concluded that clinical parameters are not correlated significantly to
DSCSA $^{17}$. While, Ogikubo et al. showed that the DSCSA was found closely related to the preoperative walking ability, leg and back pain, and health-related quality of life, and it was the only 1 previous study, to our knowledge, showed a significant positive correlation between preoperative DSCSA and clinical symptom ${ }^{13}$. Despite most of studies showed no significant correlation of DSCSA obtained by conventional MRI with clinical symptoms, Kanno et al. reported that DSCSA in axial loaded preoperative MRI significantly correlated with the severity of symptoms, and thus, MRI with axial loading provides more valuable information than the conventional MRI for assessing patients with DLSS ${ }^{8)}$. Our study, using DSCSA obtained by conventional MRI, also demonstrated no significant correlation between preoperative DSCSA and severity of symptoms, measured by VAS $(\mathrm{p}=0.234)$ and ODI $(\mathrm{p}=0.410)$.

There are some reports in the literature on serial changes of DSCSA after posterior decompression for DLSS ${ }^{6,12,22)}$. Hiriki et al. measured DSCSA preoperatively and in the early and late phases after various type of lumbar decompression surgery ${ }^{12)}$. They demonstrated that the DSCSA increased significantly between the early and late postoperative phases, but comparison of clinical result based on radiologic result was absent. In our study, the mean expansion ratio of DSCSA after ULBD was $203.7 \pm 147.2 \%$ (range $-32.9-826.1 \%$ ) and it was statistically significant $(\mathrm{p}=0.000)$.

Only a few studies have documented the correlation between the serial change of DSCSA after posterior decompression for DLSS and clinical results, and no reports after ULBD ever. Unlike the poor correlation between preoperative DSCSA and clinical symptoms documented in previous articles, serial change of DSCSA after decompression and clinical symptom showed significant correlation in these articles. Hermansem et al. reported a significant correlation between the expansion ratio of DSCSA and the patient self reported effect of surgery after laminarthrectomy for DLSS (Pearson -3.71; $\mathrm{p}=0.006$ ) ${ }^{6}$. Yamazaki et al. performed prospective study to evaluate the serial changes in clinical result and DSCSA on MRI after bilateral fenestration $^{22)}$. They reported satisfactory clinical result and sufficient expansion of DSCSA after surgery, and poor dural expansion is a factors associated with poor outcomes. In our study, both DSCSA and clinical parameters were significantly improved after ULBD, however, the expansion ratio of DSCSA was not correlated significantly with clinical outcomes, measured by VAS and ODI.

Our study has several limitations. In our database, based on medical chart, VAS score was not divided into back and leg pain. Second, our study has only immediate postoperative DSCSA without follow-up. Last, despite the facet joint level at each intervertebral space is the most commonly involved in DLSS ${ }^{15,16}$, only DSCSA could not reflect lateral recess stenosis. 


\section{CONCLUSION}

In conclusion, data collected and analyzed in the current study demonstrated significant expansion of DSCSA and improvement of clinical outcomes after ULBD in patients with DLSS, but both preoperative DSCSA and the expansion ratio of DSCSA were not statistically significantly correlated with clinical symptoms. Our result indicates that the DSCSA itself has a definite limitation to be correlated to the clinical symptoms. It also emphasizes that DLSS is a clinico-radiological syndrome, and only the narrowing of spinal canal on MRI imaging itself is not a unique tool for the determination of surgical treatment without meticulous correlation with the clinical presentation.

\section{REFERENCES}

1. Amundsen T, Weber H, Nordal HJ, Magnaes B, Abdelnoor M, et al: Lumbar spinal stenosis: conservative or surgical management? A prospective 10-year study. Spine 25:1424-1435, 2000

2. Atlas SJ, Keller RB, Robson D, Deyo RA, Singer DE: Surgical and nonsurgical management of lumbar spinal stenosis: four-year outcomes from the main lumbar spine study. Spine 25:556-562, 2000

3. Cavuşoğlu H, Kaya RA, Türkmenoglu ON, Tuncer C, Colak I, et al: Midterm outcome after unilateral approach for bilateral decompression of lumbar spinal stenosis: 5-year prospective study. Eur Spine J 16:2133-2142, 2007

4. Ciol MA, Deyo RA, Howell E, Kreif S: An assessment of surgery for spinal stenosis: time trends, geographic variations, complications, and reoperations. J Am Geriatr Soc 44:285-290, 1996

5. Costa F, Sassi M, Cardia A, Ortolina A, De Santis A, et al: Degenerative lumbar spinal stenosis: analysis of results in a series of 374 patients treated with unilateral laminotomy for bilateral decompression. J Neurosurg Spine 7:579-586, 2007

6. Hermansen E, Moen G, Barstad J, Birketvedt R, Indrekvam K: Laminarthrectomy as a surgical approach for decompressing the spinal canal: assessment of preoperative versus postoperative dural sac cross-sectional area. Eur Spine J 22(8):1913-1919, 2013

7. Hong SW, Choi KY, Anh Y, Baek OK, Wang JC, et al: A comparison of unilateral and bilateral laminotomies for decompression of L4-5 spinal stenosis. Spine 36:E172-178, 2011

8. Kanno H, Ozawa H, Koizumi Y, Morozumi N, Aizawa T, et al: Dynamic change of dural sac cross-sectional area in axial loaded magnetic resonance imaging correlates with the severity of clinical symptoms in patients with lumbar spinal canal stenosis. Spine 37:207-213, 2012

9. Lohman CM, Tallroth K, Kettunen JA, Lindgren KA: Comparison of radiologic signs and clinical symptoms of spinal stenosis. Spine 31:1834-1840, 2006
10. Moon ES, Kim HS, Park JO: Comparison of the predictive value of myelography, computed tomography, and MRI on the treadmill test in lumbar spinal stenosis. Yonsei Med J 46:806-811, 2005

11. Morgalla MH, Noak N, Merkle M, Tatagiba MS: Lumbar spinal stenosis in elderly patients: is a unilateral microsurgical approach sufficient for decompression? J Neurosurg Spine 14:305-312, 2011

12. Oba H, Takahashi J, Futatsugi T, Mogami Y, Shibata S, et al: Study of dural sac cross-sectional area in early and late phase after lumbar decompression surgery. Spine J 13(9):1088-1094, 2013

13. Ogikubo O, Forsberg L, Hansson T: The relationship between the cross-sectional area of the cauda equine and preoperative symptoms in central lumbar spinal stenosis. Spine 32:1423-1428, 2007

14. Park YJ, Park KH, Kim TW, Kim JC: Unilateral laminectomy and bilateral decompression for lumbar spinal stenosis with microscope and tubular retractor system. Korean J Spine 5(2): 77-82, 2008

15. Schonstrom NS, Bolender NF, Spengler DM: The patho-morphology of spinal stenosis as seen on CT scans of the lumbar spine. Spine 10:806-811, 1985

16. Schonstrom N, Hansson T: Pressure changes following constriction of the cauda equine. An experimental study in situ. Spine 13:385-388, 1988

17. Sigmundsson FG, Kang XP, Jönsson B, Strömqvist B: Correlation between disability and MRI findings in lumbar spinal stenosis: a prospective study of 109 patiens operated on by decompression. Acta Orphop 82:204-210, 2011

18. Sirvanci M, Bhatia M, Ganiyusufoglu KA: Degenerative lumbar spinal stenosis: correlation with Oswestry Disability Index and MR imaging. Eur Spine J 17:679-685, 2008

19. Spetzger U, Bertalanffy H, Naujokat C, Von Keyserlingk DG, Gilsbach JM: Unilateral laminotomy for bilateral decompression of lumbar spinal stenosis. Part I: Anatomical and surgical considerations. Acta Neurochir (Wien) 139:392-396, 1997

20. Spetzger U, Bertalanffy H, Reinges MH, Gilasbach JM: Unilateral laminotomy for bilateral decompression of lumbar spinal stenosis. Part II : Clinical experiences. Acta Neurochir (Wien) 139:397-403, 1997

21. Thomé C, Zevgaridis D, Leheta O, Bäzner H, Pöckler-Schöniger $\mathrm{C}$, et al: Outcome after less-invasive decompression of lumbar spinal stenosis: a randomized comparison of unilateral laminotomy, bilateral laminotomy, and laminectomy. J Neurosurg Spine 3:129-141, 2005

22. Yamazaki K, Yosida S, Ito T, Toba T, Kato S, et al: Postoperative outcome of lumbar spinal canal stenosis after fenestration: correlation with changes in intradural and extradural tube on magnetic resonance imaging. Journal of Orthopaedic Surgery 10(2): 136-143, 2002

23. Zeifang F, Schiltenwolf M, Abel R: Gait analysis does not correlate with clinical and MR imaging parameters in patients with symptomatic lumbar spinal stenosis. BMC Musculoskelet Disord 9:89, 2008 